\title{
Biomimetic Applications of Mimosa pudica L. in the Theoretical Development of a Pneumatic Actuator
}

\author{
Gabriel Yuto Munakata ${ }^{1,2}$ \\ https://orcid.org/0000-0001-6161-0353 \\ Plínio Rodrigues de Oliveira Zanini, ${ }^{1,2}$ \\ https://orcid.org/0000-0001-5142-2674
}

Silvia Titotto ${ }^{1,2^{*}}$

https://orcid.org/0000-0002-1089-6969

\begin{abstract}
${ }^{1}$ Federal University of $A B C$, Center of Engineering, Modeling and Applied Social Sciences (CECS), Santo André, São Paulo, Brazil; ${ }^{2} 4 \mathrm{D}$ Printing and Biomimetics (4DB) Research Group, Brazilian National Council for Scientific and Technological Development (CNPq) Directory, Brazil.
\end{abstract}

Editor-in-Chief: Alexandre Rasi Aoki

Associate Editor: Adriel Ferreira da Fonseca

Received: 2020.07.09; Accepted: 2021.07.26.

*Correspondence: silvia.titotto@ufabc.edu.br; Tel.: +55-11-49968246 (S.T.).

\section{HIGHLIGHTS}

- $\quad$ The movement of Mimosa pudica L. has potential for the use in formulation of actuators.

- $\quad$ The developed actuator has the potential to be used in the automation area.

- Using simple materials is an interesting proposal to be explored in bioinspired actuators.

Abstract: For years, plants have tried to adapt to the environmental changes caused by time, improving and developing their biological structures. Many of these structural and functional properties of plants have great potential for the development of concepts in the field of biomimetics. Recent previous studies have shown that the movement of Mimosa pudica L. is caused by the variation of turgor pressure within the cells of organs motor, that is, the influx and efflux of water by osmosis, generating reversible changes in the shape of the plant. Thus, this article sought, through research and literature references, to carry out a survey of studies related to the seismonastic movements of the plant and its applications in the design of technological innovations. In addition, it presents the development of a pneumatic actuator based on the abstraction of the morphology of the primary pulvinus of the plant and the concept of bioinspired design of the theoretical model based on the technology of soft robots. As a result, the bioinspired actuator model of the plant movement is described. In addition, with a simulation, it was possible to observe that the flexible modules are capable of generating the proposed movement and allow movement of the actuator. With the study, it was possible to understand that the movement of the plant appears as an embryo for the projection of technologies, and that the proposed study appears as the basis for research with pneumatic actuators.

Keywords: Mimosa pudica L.; bioinspiration; biomimetics; pneumatics; soft robots. 


\section{INTRODUCTION}

Plants have developed a multitude of mechanisms for the action and movement of their organs to such an extent that knowledge related to biological mechanisms is based on inputs for the projection of adaptive structures and intelligent materials [1,2]. The field of intelligent botanical structures is an area of interest to be explored [3] because nature, for thousands of years, has sought to improve the functioning of its structures amid complex and competitive conditions [2]. Thus, their movements emerge as derivable models for conception [4] in biomimetic applications in order to allow the construction of effective technological innovations.

In the midst of a wide field of biological varieties, the authors find a species capable of performing a specific movement conditioned by external mechanical stimuli, Mimosa pudica L., popularly known as nonsense or no-me-touch [5]. It is a plant belonging to the legume family [6], capable of responding with rapid movements, which can be divided into three stages: perception of the stimulus, transmission of electrical signals and mechanical, hydrodynamic and biochemical induction [7].

One of the characteristics responsible for allowing the movement of the plant is the turgor mechanism. This phenomenon is triggered by the variation of the turgor pressure within the cells of the pulvinus of Mimosa pudica L., that is, the pressure caused by the entry of water into the cell by osmosis. This allows balance with the hydrostatic pressure that is exerted on the cell wall [8].

In this article, the main focus is on a description of the structures integrating the Mimosa pudica L. plant and the mechanism involved in the seismonastic movement, summarizing current concepts and recent discoveries about their reaction to external mechanical stimuli. Furthermore, a model of pneumatic actuator modeled in the SolidWorks software is presented. This model was obtained through the abstraction of the concepts used in the movement and the morphological structures of the plant, in addition to the application of studies related to soft robots. The first part of the article provides an insight into the theoretical fundaments of the main structural components of the plant and its movement mechanism on top of some technological applications that employ studies related to Mimosa pudica L. The second part describes the methodology and materials used in the development of the actuator as well presenting the abstraction process used for the conception of bioinspired design and the studies that served as a basis and inspiration for the projection of the pneumatic actuator model. The final part is dedicated to discussing the results obtained in this research describing the final conception and simulation of the theoretical model.

\section{Theoretical fundaments}

\section{Morphological structures}

Before understanding the mechanism and functioning of Mimosa pudica L., it is important to observe and analyze its biological structures. The plant is characterized as a small and prostrate shrub, reaching a height of one meter and more than two meters in horizontal extension [5]. Its roots are cylindrical in a brownish-gray tone, with a thickness varying up to $2 \mathrm{~cm}$ in length [9]. Its stems are cylindrical (up to $2,5 \mathrm{~cm}$ in diameter), reddish-brown and sparsely or densely armed with curved spines - which have branches called petioles that are connected to the stems utilizing the primary or main pulvinus $[5,9,10]$. At the other end, between the spines (thin and flexible branches) and the petiole, the authors find the sub-pulvinus (secondary pulvinus), which commonly controls the orientation of the leaves towards the source of irradiating light and supports one or two pairs of rachis, which house - by means of the tertiary pulvinus (or pulvinulo) - between fifteen to twenty-five pairs of oblong leaflets from three to twelve millimeters in length in a bi-pin pattern [5, 10, 11, 12]. During the day, the leaves are spread out, and during the night they curve downward. In addition, when stimulated by external factors, their leaflets bend and their petiole curves, reclining their initial positions after about 30 minutes [11]. 


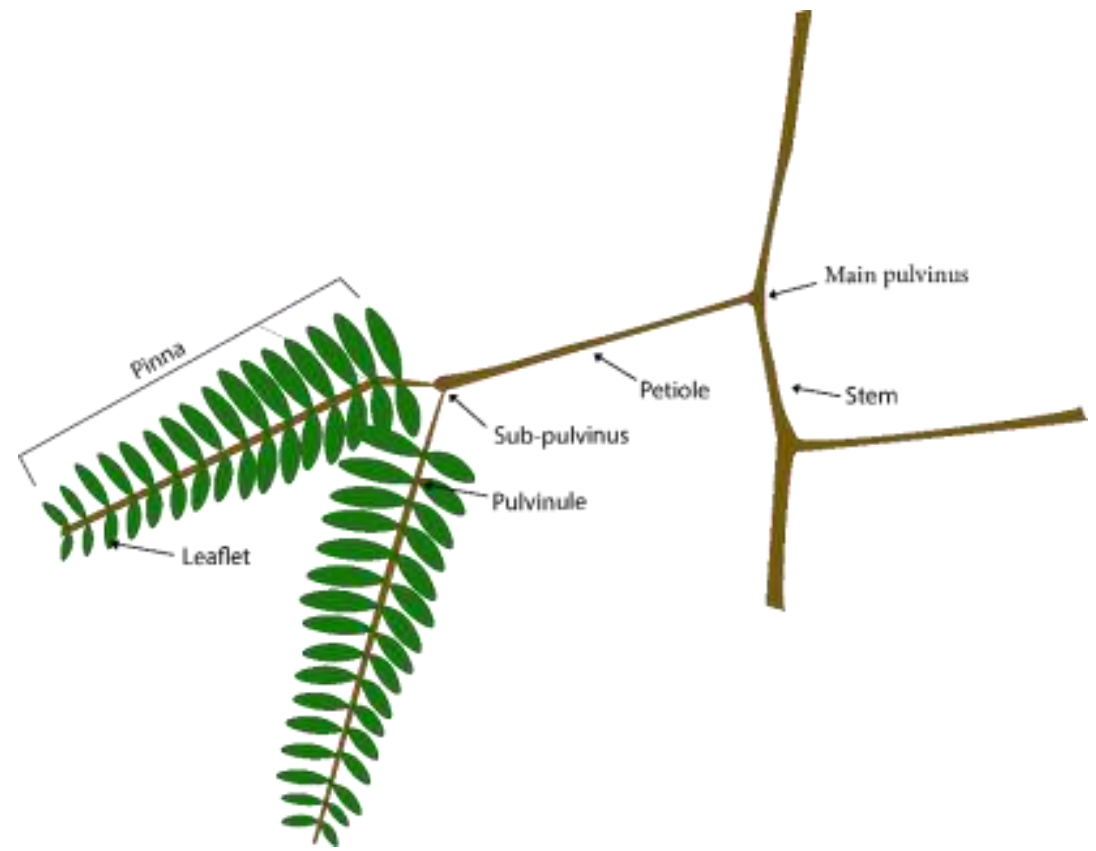

Figure 1. Representation of the morphological structure of Mimosa pudica L. (Adapted from [2]).

\section{Cell organization}

Anatomically, the pulvinus of Mimosa pudica L. are characterized by the presence of specialized motor organs. Such organs allow descending (primary and secondary pulvinus) and ascending (tertiary pulvinus) movements in the plant's structures [11,13]. Three vascular bundles of the stem join at the entrance of the primary pulvinus to form a single median bundle surrounded by about 12 layers of cells in the cortical parenchyma [10]. This nucleus is surrounded by three regions of cells, the first being characterized by cells of the endodermis, which do not interfere in the pulvinar movement, only assist in the perception of gravity. The second region consists of cortical parenchymal cells. These cells have vacuole and cytoplasm. The third layer is formed by all the cells in the cortical parenchyma - diametrically larger than those in the second region. These cells have vacuoles and thin cytoplasmic walls, abundant cytoplasm, vacuoles and intercellular gaps $[11,13]$. In general, the sensor-motor parenchyma can be divided into two regions, the upper cortex (adaxial) and the lower cortex (abaxial). Such regions undergo pressure variation within the cells and cause the petiole to deflect $[14,15]$.

\section{Movement mechanism}

A striking feature of the plant refers to the behavior of its rapid movement when stimulated by external mechanisms such as touch, wind, light or vibration. These stimuli drive the mechanism of opening and closing the leaves and folding the pulvinus, which is sufficiently flexible and capable of moving the petiole omnidirectionally [16]. Responsive movements are generated by the alteration of turgor in the cortical cells of the primary pulvinus, secondary pulvinus and tertiary pulvinus, which are responsible, respectively, for the movement and orientation of the petiole, leaves and leaflets [17, 18]. Thus, the hydraulic movement of Mimosa pudica L., which is more efficient in relation to other plants, gives it an important and necessary characteristic for the movement of its organs [12].

The mechanism of action caused by the variation of turgor occurs by the change of pressure within the cells of the Mimosa pudica L. pulvinus [8]. This pressure is caused by the entry of water into the cell by osmosis and allows balance with the hydrostatic pressure that is exerted on the cell wall [8]. Anatomically, the pulvinus consist of water-conducting vessels (xylem). These have thick and lignified cell walls, surrounded by thin-walled motor cells that can suffer visible swelling and shrinkage $[3,12]$.

Water is transported axially through xylem vessels over long distances and is distributed radially between adjacent tissues [12]. When stimulated, the plant redistributes the water flow between the cells of the primary pulvinus, allowing the movement of the leaves to occur due to an increase in pressure in the upper (adaxial) cells, and the sudden decrease or loss of turgor in the lower motor cells (abaxial), where the vacuoles contract due to stimulation - presumably caused by the loss of salts in the cell, and the expansion or recovery is caused by the absorption of fluid rich in salts by the vacuole.

In other words, ionic salt exchanges occur between the intracellular and extracellular medium under stimulation $[5,12,14]$. According to the research presented in [3], the lifting of the leaves is triggered by an 
increase in the pressure and volume of the motor cells due to the absorption of $\mathrm{K}+$ ions in the extending cells, so that when the $\mathrm{K}+$ channels in the extending cells close, the channels in the flexor cells open and release the pressure from the turgor, causing the joint to lose its stiffness and let the leaf fall.

\section{Examples of biomimetic applications of the plant}

The plant's ability to respond with rapid movements when subjected to external stimuli has attracted the interest of researchers and engineers for the biomimetic conception in the construction of bioinspired structures [18]. Mimosa pudica L., in addition to having simple movements and geometrically measurable motor organs, has a great exploitable potential that supports research and studies on the development of biological actuators in biomechanical systems [19, 20].

Among the fields of application of the principles of plant movement, the authors have the development of "soft materials", materials whose properties are related to a strong reaction to weak stimuli, self-assembly and non-linear responses [20]. Thus, an applicable example of these principles is the "Mimosa origami", a nanostructured regime capable of self-organizing from a small stimulus, based on the rational design and self-organization of Mimosa pudica L. [22]. In the "Mimosa origami", the polymeric material, consisting of layers of paper, PVP (Polyvinylpyrrolidone), PCL (Polycaprolactone) and PVC (Polyvinyl chloride), has two regions - one hydrophilic and the other hydrophobic - which react in different ways in contact with the water, so that, when stimulated by a drop, it transforms surface energy into directional kinetic and elastic energy [22].

Similarly, polymeric microactuators with microfluidic channels triggered by swelling have been developed and implanted in structures that seek, for example, to mimic the movement of the plant. These microactuators were manufactured using projection micro-stereolithography (3D micro-fabrication technique) - of photocurable polymers that respond with swelling of specific regions to provide the movement of the structure [23].

Another example of the application of sensory principles can be seen in paper actuators, the Printed Paper Actuator. It is a composition formed by a substrate (paper, plastic films, among others) and a thermoplastic layer (e.g. graphene-based PLA), built at a 3D modeling printer (FDM) and which responds with flexible movements due to the heating system effect by electric current in the PLA [24]. Thus, the material contracts and relaxes, causing the system to move. Among the possible applications is the artificial control of the movement of Mimosa pudica L., which responds with a sequential activation of the folding of the leaves when touched, one after the other [24].

Thus, the plant has a characteristic movement that has inspired work in the most diverse interdisciplinary areas within the scope of science. Such examples show the various possibilities of applying bioinspired concepts in the construction of new technologies. Similarly, this article sought in the various scientific examples based on the movement of the plant, to formulate the construction of a bioinspired technology related to the area of pneumatic actuators. For that, it also used the concepts of soft robots and technologies developed based on this interdisciplinary science.

\section{Soft robots and Mimosa pudica $L$.}

Soft robots appear as an innovation for the development of a control mechanism capable of dealing with unexpected situations in unstructured environments. It is a soft robotics that seeks to enable robots with resilient, low-cost and maneuverable mechanisms, which are not based on control systems, but on the properties of the material and on the morphology of their body structures, in order to explore their own "mechanical intelligence" of soft materials [25, 26]. An application example is the development of OctArms robots that seek to imitate the complexity of the movement of cephalopods (squid, octopus). Thus, they use pneumatic muscles to move their structures - composed of silicone elastomers - through pressurization of air or fluid [25].

The importance of soft components in natural organisms seems to have great relevance to reducing the complexity of controlling their structures. Several organisms perform their actions based on soft systems and materials, such as caterpillars, octopuses, birds and plants [27]. Among the advantages, soft robots are able to adapt to uncertain and complex environments and interact more safely in operations with human beings $[28,29]$.

Thus, based on systems of soft robots, seismonastic movements of Mimosa pudica L. could be structured in order to make fluid pressurization technology compatible with the variation of turgor within the pulvinus motor cells. Thus, taking as an example the tetrapods - robots built from lithography, soft materials and which move from pneumatic actuation [30] - it is possible to develop an actuator design based on the plant's movements from fluid channels that interact with the system. In addition, studies based on the actuator in honeycomb pneumatic networks (HPN) and the caterpillar movements serve as great inspiration for the design of an actuator design, as detailed in section materials and methods. 


\section{MATERIALS AND METHODS}

Considering the morphological structures and the movement of Mimosa pudica L., and also using the concepts used by soft robots, the procedures for the construction of a theoretical actuator were carried out. The model used the concepts of biomimetics so that its movement could be controlled with pneumatic technology from the modification of soft materials. Therefore, for the construction of the model, bioinspired designs were used as materials for the methodology to enable the simulation of the plant's movement. The models were built in 3D using the SolidWorks software, with the final design presented in the results. Design studies are presented below:

\section{Modeling process of the pneumatic actuator}

From an analysis of the morphological structure of the organ responsible for the movement in the plant (primary pulvinus), the authors used an abstraction process to recreate it in technological applications. The main aspects considered were related to the movement of the structure based on the plant's capacity to undergo the process of turgor of its motor cells. Firstly, the behavior of the plant and the morphological structure of its primary pulvinus were observed, and from the analysis, the procedure for building drawings was initiated to simplify the structural pattern when the movement of the plant modifies the organization of its motor organ cells.

Based on the studies, it was observed that the behavior of the structure, when undergoing the curvature process, could be simplified as a rule of geometric shapes. Thus, the first process to abstract the concept used by the plant was through a rectangle pattern. When curved, they could be characterized as triangles centered with one of its vertices in the center of a circumference (Figure $2 \mathrm{~A}$ ). As the pulvinus is characterized by motor cells of the cortical parenchyma and by vessels conducting water (xylem and phloem), it is possible to perceive that its morphological organization is established in a junction of spheres that, under a growth process, trigger propagation of forces that displace the other spheres, characterizing the curvature of the plant structure. Therefore, the addition of spheres that simulate the observed behavior was used in the established pattern (Figure 2 B). In the obtained structure, the spheres perform the function of changing their volume to generate the curvature movement. In this way, the pattern for the composition of spheres was simplified, eliminating the other geometric shapes (Figure $2 \mathrm{C}$ ).

The purpose of this model is to use the concept that the spheres, when increasing their volumes, as they are organized close to each other, are able to flex the base in a curvature movement. In this way, a first model of spheres was constructed in a circular pattern that relates to the cells of the motor organ of Mimosa pudica L. (Figure $2 \mathrm{D}$ ). At the center of the structure, two distinct regions would be responsible for conducting the flow of fluid (relating to the conducting vessels). With the pressurized vessels, the fluid would flow to the internal regions of the spheres, causing them to swell and expand. Thus, it would trigger the pressing of the spheres against each other and the structure would curve downwards or upwards (depending on the pressurized region). Using the abstracted model, the study was oriented towards the development of an actuator that could, in addition to simulating the plant's behavior, present a technological application centered on biomimetic aspects. In this way, the structure of a model was built using the SolidWorks modeling software. The obtained resource can be better visualized according to Figure $2 \mathrm{E}$. 


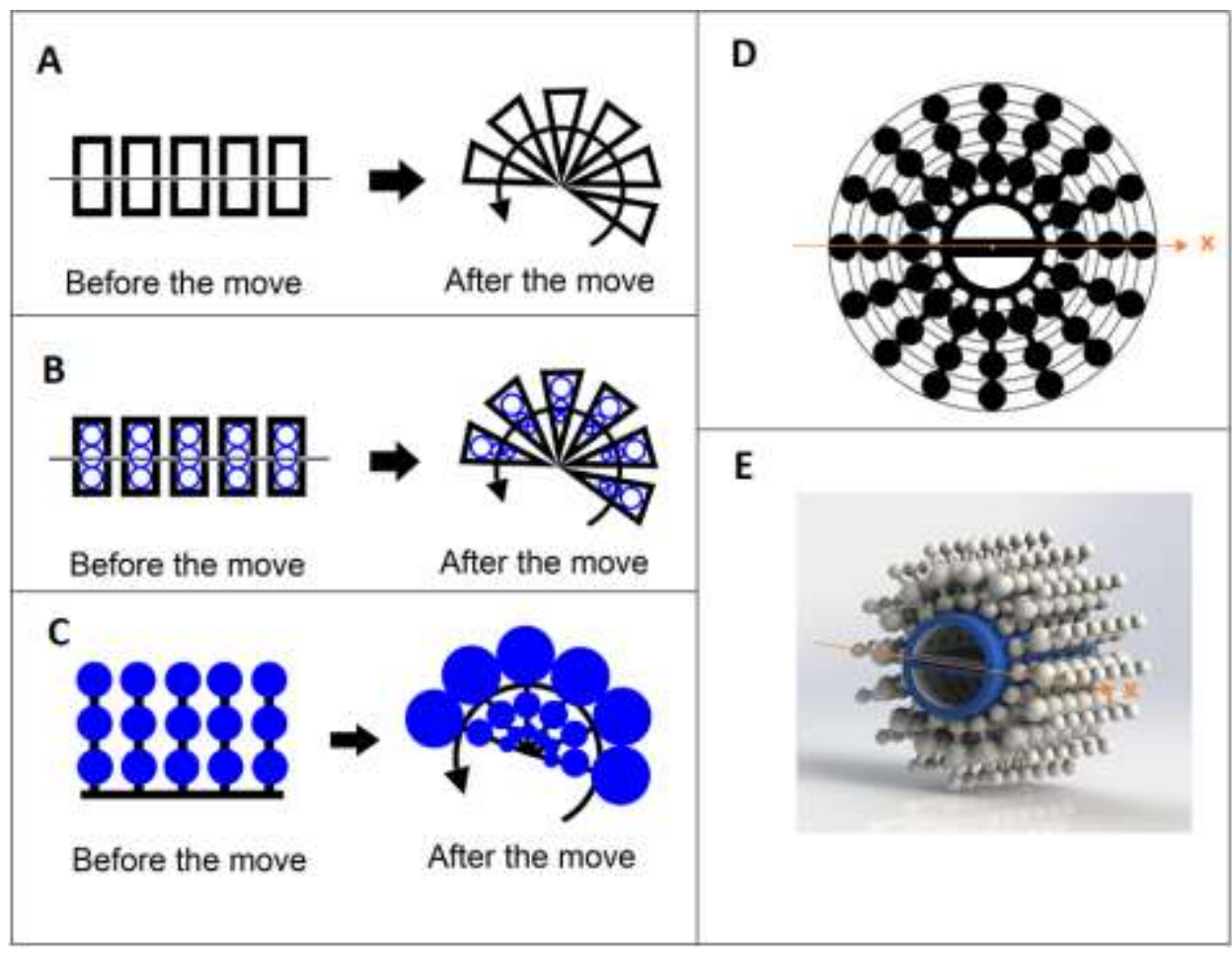

Figure 2. Process of abstraction of the morphological structure of the plant during its movement $(A-C)$ and model obtained from the drawings in the modeling step (D-E). (A) Pattern of geometric shapes according to the abstraction of the concept used by the plant. (B) Pattern of geometric shapes with the addition of spheres. (C) Pattern obtained after the structure simplification process. (D) Sphere pattern in a circular arrangement. (E) 3D representation of the model of the first actuator developed in SolidWorks.

However, due to the complexity of construction in equipment such as 3D printers, it was necessary to reevaluate the structure and look for simpler and moldable patterns. Analyzing the first actuator, an interesting feature observed is that the movement of the structure was related to specific regions - as can also be seen in the behavior of Mimosa pudica L. Therefore, these regions were separated by means of rectangles, which allowed the formulation of a new construction of the model. The use of rectangles was chosen because it is a simple way of dividing the structure and taking advantage of the two regions of action for the flexion and deflection movement. The procedure can be seen in Figure $3 \mathrm{~A}$.

Based on the separation, one of the aspects observed is that the structure could be better used and it would also be easier to build an actuator if it was possible to structure it with plug-in modules. Therefore, the research was oriented towards the construction of a simpler model that could be assembled with different parts and built modularly. For that, the Reconfigurable Omnidirectional Soft Robot presented in [29] was used as inspiration in soft robots. These soft omnidirectional robots - inspired by the locomotion of caterpillars are structured in a pneumatically reconfigurable mechanism - which is composed of nine modules arranged in a 3×3 matrix and capable of moving in three degrees of freedom in one plane, being reassembled and exploring non-standard environments structured [29]. Such a robot is able to move from the pressurization and depressurization of its modules, responding with different types of movement from a pneumatic control.

One of the characteristics of the soft robot is its construction based on a modularization strategy. This aspect has advantages over structures built continuously, such as, for example, lower financial costs (only defective modules must be replaced), lower time costs (new features can be achieved by combining materials, sizes and structures) and better adaptability (grouping modules for different environments and tasks) [29]. Thus, the robot can recombine with other modules from a system of neodymium magnets and with correct orientation.

Based on the modular concept, the actuator employed these characteristics in order to allow the construction of a structure that could be developed separately and joined by fitting parts, ensuring flexibility in the construction of the system and allowing replacement of parts in case of damage or component failures. The model presented in [29], by using the features of soft robotics and being structured with plug-in modules, allows flexibility to build each feature that makes up the robot separately.

This aspect applied to the proposed actuator model, in addition to facilitating the construction process through the 3D printer, also allows for simpler adjustments without the need to remodel the entire structure. For this, the geometric shapes that represent the new actuator model were used. Analyzing the prototype, two regions were defined (above and below of the square in Figure $3 \mathrm{~A}$ - regions in blue), which are related 
to the active components that suffer from the swelling process. The square provides stability in the structure (in yellow in Figure $3 \mathrm{~A}$ ). The central rectangles (in green in Figure $3 \mathrm{~A}$ ) refer to the flow-conducting vessels. With the separate modules, it was possible to build a new actuator model from the junction of the other modules. One aspect of the model is that the inflatable chambers, when undergoing the swelling process, force the other modules to move away, triggering a process of propagation of movement (as the cells of the plant force each other). The schematic of the model is shown in Figure $3 \mathrm{~B}$. The fluid channels and inflatable chambers are indicated in the Figure 3 by the blue arrows.

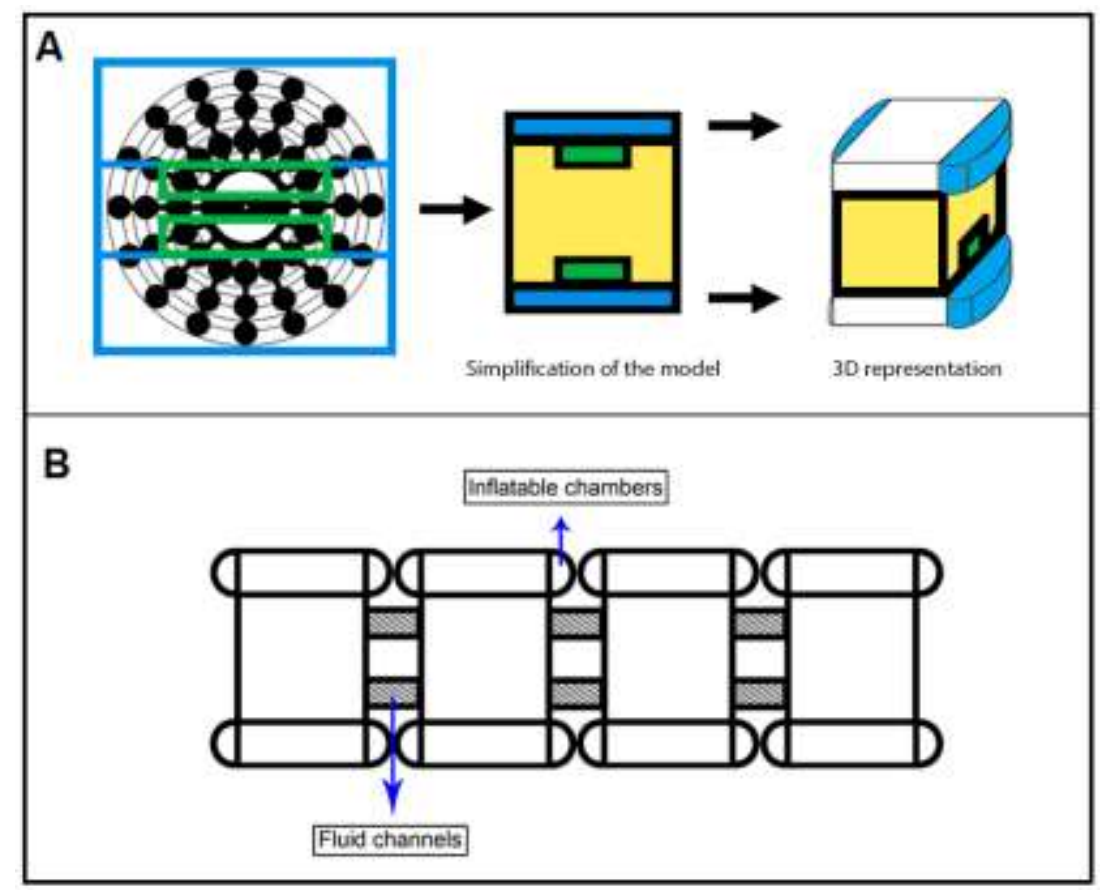

Figure 3. Simplification process of the initial structure $(A)$ and representation of models for the composition of the new actuator (B). (A) Division of the regions active in the movement process and the new model obtained from the simplification. The square (in yellow) represents the support structure. The green rectangles are the pressurized air transport channels and the regions above and below the structure (in blue) are the structures that act to trigger the movement. (B) Representation of the junction of the modules, indicating the position inflatable chambers and fluid channels (blue arrows).

Moreover, the construction of chambers could be improved so that it could undergo elastic deformation and guarantee stability in the structure. With this in mind, studies of the soft manipulator based on honeycomb pneumatic networks (HPN) developed by the research presented in [28] were used. It is a soft structure capable of achieving a balance between flexibility and load capacity, maintaining its strength even under collision or pressure [28]. Honeycomb pneumatic networks use an incorporated pneumatic mesh capable of deforming the structure, allowing it to present properties such as elongation rate and crush resistance [28]. Structurally, the manipulator is made up of hexagonal chambers whose geometric shape is modified from the internal pneumatic network in the form of air pockets, which, when inflated, allow the movement of the structure without losing structural resistance and stability [28].

Thus, the integration of the concepts involved with the abstraction of the cellular structure of the cortical parenchyma of the plant and of the HPN allows the formulation of a mechanism that employs the concepts of flexibility and resistance, in addition to allowing the movement of the structure in a controlled manner. Thus, the inflatable chamber of the actuator developed in this study was based on both concepts, integrating the behavior of the plant and structuring the chamber in closed hexagonal networks, capable of undergoing non-destructive deformation and allowing the movement of the actuator. With that, an internal structure of the inflatable chamber was developed as can be seen in Figure 4. 


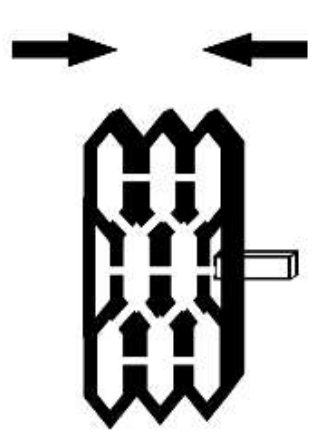

Contracted chamber

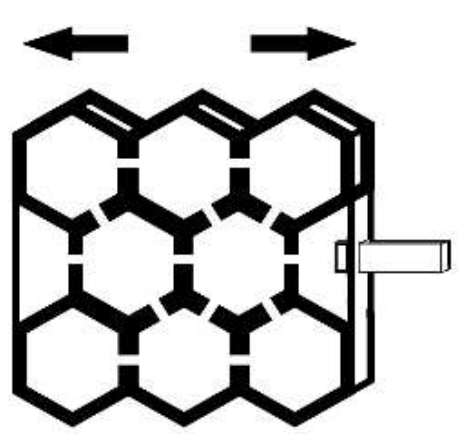

Relaxed chamber

Figure 4. Representation of the internal structure of the inflatable chamber obtained from the bioinspiration of the morphology of the primary pulvinus of the plant and of the concepts presented in honeycomb pneumatic networks (HPN). Following, the process of contraction and relaxation of the chamber.

The inflatable chamber plays the role of the cells that undergo turgor in the plant. Each hexagon represents a cell that, when swelled, triggers a reaction that forces each to expand or contract. It was preferred to work with hexagons instead of spheres not only for the ease of designing the model, but also for the aspects of resistance and stability, as shown in honeycomb structures [28].

\section{Construction of the new model}

Considering the study of the bioinspiration design process, a new model was built in SolidWorks. The model is organized in modular structures, which can be done using 3D printing and casting with soft materials, such as Smooth-On Ecoflex silicone and Wacker Chemie Elastosil M4601 silicone [31]. Regarding the flexible module, its movement occurs from the pressurization of chambers in hexagonal format capable of altering its geometric shape without causing major deformations that wear out the material. In addition, the actuator structure is composed as a support for the flexible module and the air conducting tubes.

All parts were developed using the SolidWorks software. They were modeled with the modular design as a goal, enabling their easy assembly and possible replacements in case of damage to one of the parts. The main structures developed are the flexible modules inspired by the cellular organization of the plant and the concepts involved with HPN. As a way of connecting the structures, easy-fit pins were designed. They are pieces capable of connecting both the pipes and the supports and the inflatable chambers. They are inserted into the holes in the soft material structure and connect the components in order to prevent certain regions from suffering possible leaks.

The pins are different due to their positions in the structure. The open pins are responsible for connecting the pipes to the respective flexible modules. Therefore, there is a need for channels for fluid air transport. However, the closed pins are used only to connect the inflatable chamber to the support, and thus allow the structure to interact together. In addition, as its function is to connect the structures, its materials are not soft, that is, they could be made from hard polymers such as polylactic acid (PLA), ABS or other 3D printing materials. Finally, the support was designed to allow the connection with the inflatable chambers and the passage of the pipes to organize the channels.

Figure 5 shows the design of the developed concept, where it is possible to visualize the side view of the actuator $(A)$, the side view in section $(B)$ and the 3D representation $(C)$. In Figure $5 \mathrm{C}$, the black arrows indicate the parts of the actuator. The pins are the structures in blue, the brackets with holes for fitting the pipes and actuators the parts in gray, and the flexible module the structure in white. For the full-scale projection, it was necessary to remodel the pieces with their respective sizes. Therefore, based on the second model, the structure for a full-scale projection was developed, so that the parts were dimensioned according to the construction of a prototype that could simulate the behavior and be made using 3D printing and soft materials. 


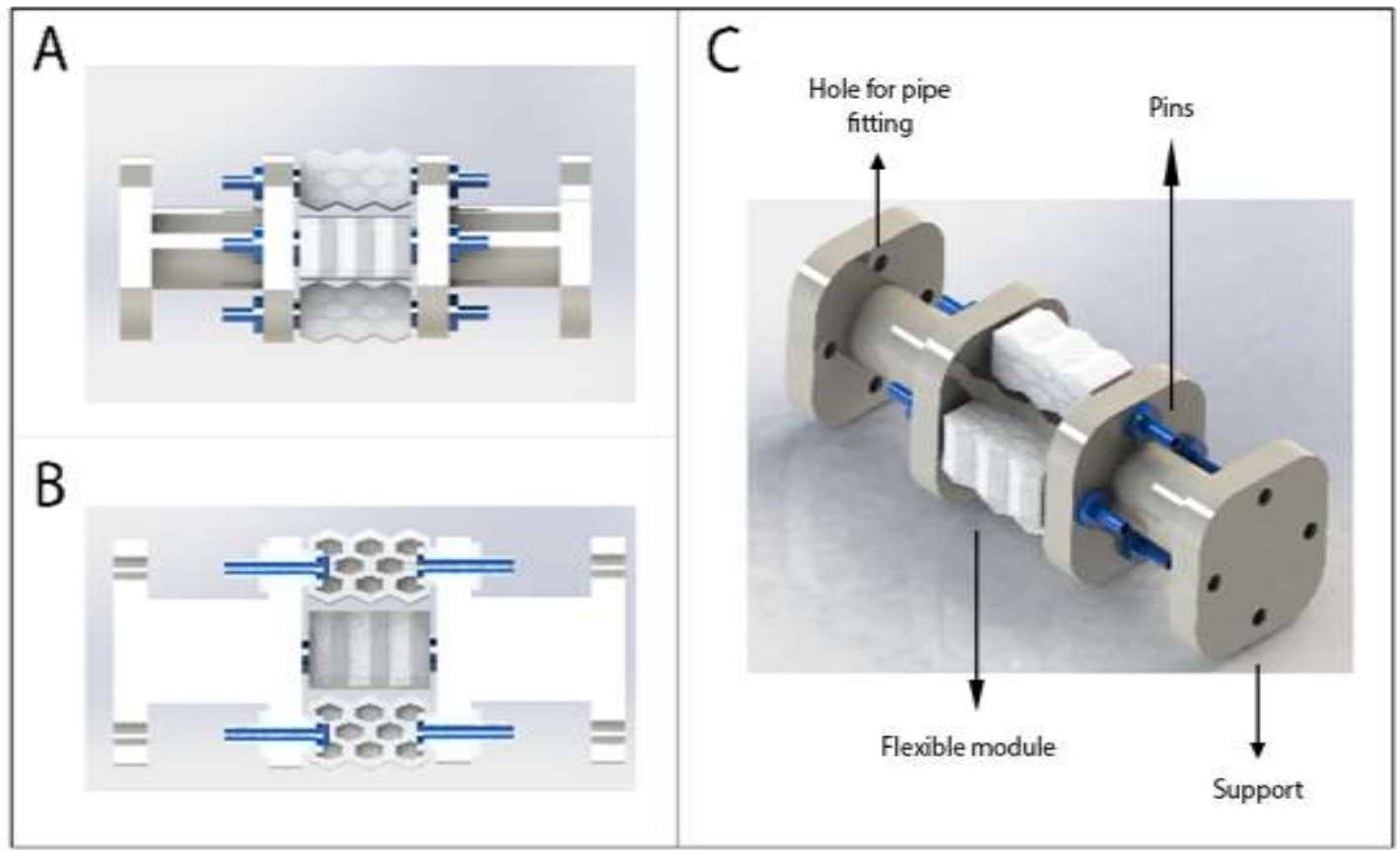

Figure 5. Actuator model designed in full scale. (A) Side view. (B) Sectional view. (C) 3D representation with black arrows indicating the actuator components.

In this actuator model, the main characteristic related to its movement is the joint performance of the other flexible and inflatable modules. In this way, when one of the actuators is pressurized, a horizontal expansion movement is generated, which propels the wall of the solid structure of the actuator and consequently causes the modules that are depressurized to contract. In this way, the individual performance of each module generates the joint performance of the structure, allowing flexibility in movement in different directions. In the scheme illustrated in Figure 6 below, only two flexible modules are presented to exemplify how the dynamics of movement occur with the joint performance of the other flexible structures. However, the main feature of the model is that it can generate movements in other directions depending on how the actuators are activated. The movement scheme can be better visualized according to Figure 6 .
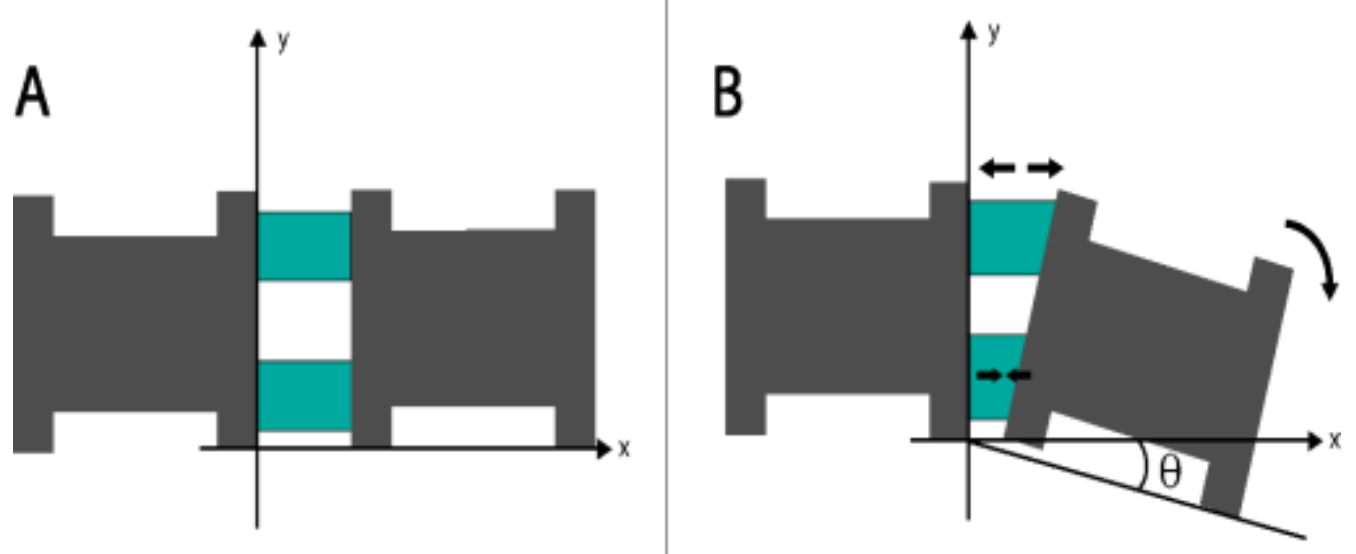

Figure 6. Movement scheme of the bioinspired actuator. The green regions indicate the position of two actuators as examples, so that when the upper actuator is pressurized, the lower actuator retracts (which is depressurized), causing a bending movement. A) Actuator before pressurization. B) Actuator after pressurizing one of the modules.

Before prototyping in a real model, a study on the dynamics of movement was performed on the Simulation Xpress platform of SolidWorks. The objective of this study was to perform a linear static simulation and understand how the internal pressure inside the flexible module walls can generate a horizontal expansion/retraction movement, generating a displacement that makes the actuator achieve its movements.

For the simulation, a pressure of $22,9 \mathrm{~Pa}$ was used on the internal walls of the model, as well as the material chosen was natural rubber from the library of the software and which approaches the study with soft materials). The material and the applied pressure value were defined to analyze the displacements and the stress distribution in the structure and allow evaluating its performance. The chosen material features a 
$10 \mathrm{kPa}$ elastic modulus, Poisson'sratio0,45, a specific mass of $960 \frac{\mathrm{kg}}{\mathrm{m}^{3}}$ and a tensile strength of $20 \mathrm{MPa}$. The results obtained with simulation are presented in the results section.

In the flexible module, as a way to understand the dynamics of movement from a simpler analysis, a study was made with only one of the inflatable chambers. In this structure, one side of the wall in a rectangular shape was fixed (left side of the shape shown in Figure 7). Internally, pressure was applied to the structure's walls to analyze the expansion and retraction movement. The sidewall opposite the fixation point was built with a greater thickness (value of $2,00 \mathrm{~mm}$ ). This aspect was necessary so that the wall opposite the fixation point would not suffer from deformations, since the purpose of the simulation was to analyze the displacement of the structure horizontally on the x-axis, that is, the result of the displacement from the internal pressurization of the model.

To observe the dimensions used for the simulation, Figure 7 is shown below. Since the loads used were constant pressures, the depth of the presented design provides no extra information, since the authors aim to demonstrate a short section and how it deforms to achieve the movement desired.

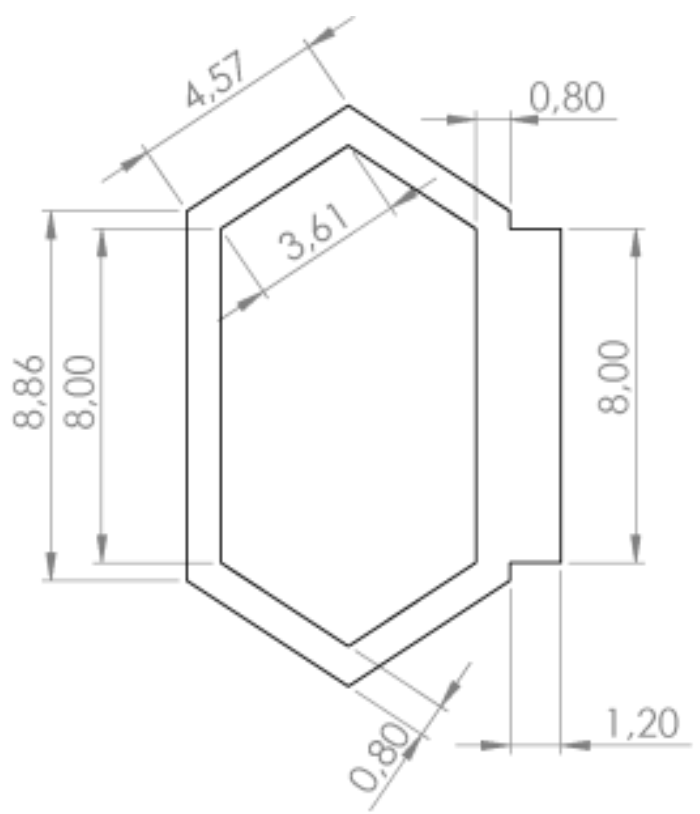

Figure 7. Dimension values of the flexible module chamber. Values shown in millimeters $(\mathrm{mm})$.

\section{RESULTS}

From the simulation, the results of two displacements were obtained. The first result is related to the application of pressure on the internal walls in order to expand the structure. The second result is related to the retraction movement so that the direction of the applied pressure was reversed. In this simulation, a qualitative study of a linear static simulation was performed to obtain a displacement of approximately $10 \%$ of the total value of the length of the chamber $(8,80 \mathrm{~mm})$, that is, a displacement of $0,88 \mathrm{~mm}$. For this, it was necessary to readjust the internal pressure values. Thus, the application of a pressure of $22,9 \mathrm{~Pa}$ obtained better approximations of the desired result. As a result of the simulation, a maximum resulting displacement (URES) of $0,8797 \mathrm{~mm}$ (regions in red shown in Figure 8) and an average displacement of $0,5865 \mathrm{~mm}$ (regions in green shown in Figure 8) was obtained. The results obtained with the simulation are shown in Figure 8. 
A

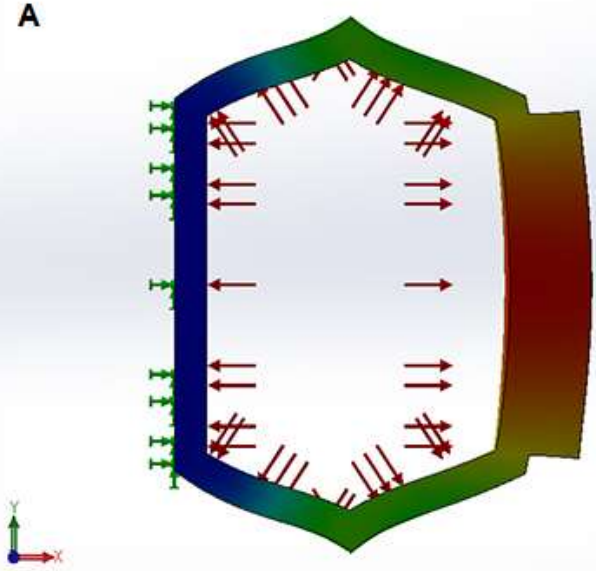

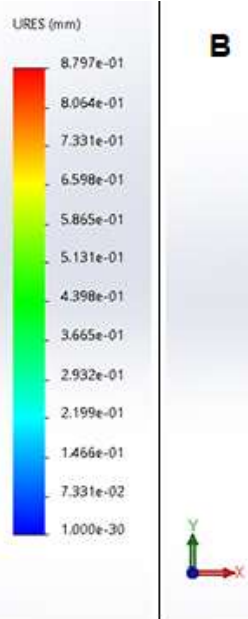

B

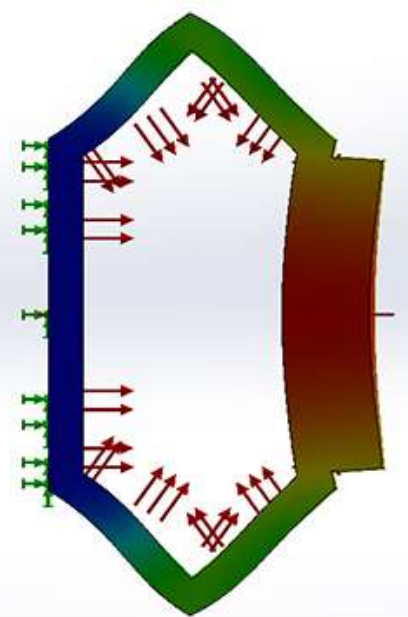

uats (men)

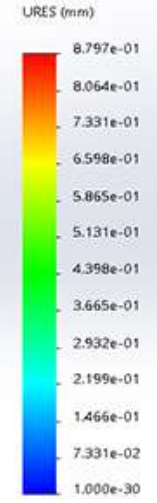

Figure 8. Linear static simulation of the flexible module chamber in SolidWorks. The green arrows indicate the fixing point. The red arrows are the pressure points. Arrows in the outward direction of the structure indicate positive pressure and arrows in the inward direction of structure the negative pressure. In the structure, the average displacements are indicated by the regions in green, while the regions in red indicate the maximum displacements. (A) Simulation of the expansion movement. (B) Simulation of the retraction movement.

As can be seen in the simulation, the result of linear displacement in the direction of the $\mathrm{x}$-axis is greater on the side opposite the fixation point. This demonstrates that when internal pressure is applied in both the expansion and retraction directions, it is possible to obtain a linear movement. Thus, in the simulation the structure is capable of carrying out the proposed movement, so that the observed behavior for a chamber can be extended to obtain a greater range of linear displacement when combining other segments of the same structure. In other words, the flexible module proposed in this study, with $n$ internal chambers would be able to perform a linear movement, and when combined with other flexible modules, it could allow the actuator to move in several directions.

In order to understand the combination of parts and observe the assembly of the actuator, the prototyping of the model proposal in real size was also carried out. For its construction, a mold (in software and in real scale) was modeled and printed for the stiffening of the flexible modules. The printed parts were made with ABS material. The full-scale project has dimensions of a total length (structure printed next to the flexible module) of $152 \mathrm{~mm}$, width of $48 \mathrm{~mm}$ and height of $48 \mathrm{~mm}$. The full-scale prototype was built as a way to analyze the proposal of bioinspired design and to understand aspects that are not possible to predict only with simulations (fitting the parts, difficulty and ease of assembly, among others). Finally, the final result obtained both in the SolidWorks software and in the project printed in 3D and modeled in full scale are presented in Figure 9.

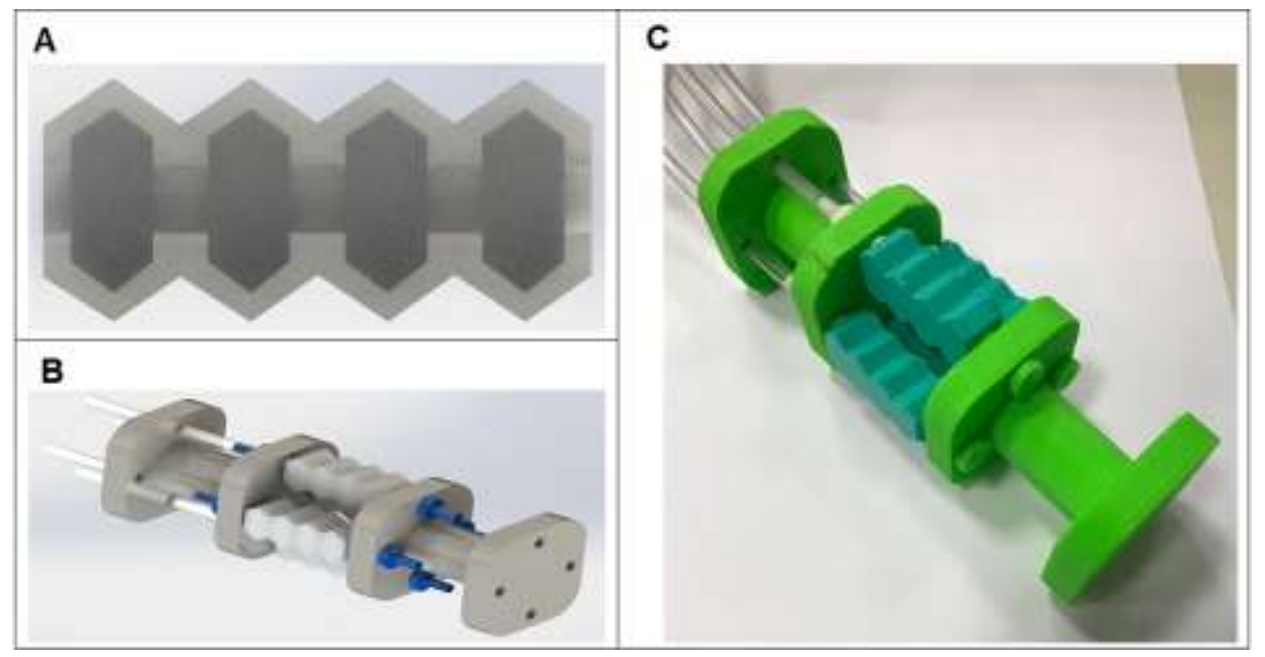

Figure 9. Final results of the modeling and the printed actuator. (A) Sectional view of the flexible module obtained from simplifying the structure in several chambers in the shape of hexagons; (B) Final actuator model designed in real sizes in SolidWorks; (C) Photo of the final actuator obtained in real size and printed on a 3D printer. 


\section{DISCUSSION}

Since the focus of the present study was to demonstrate the possibility of a design methodology, not considering the development of a specific product, and also to present the proposal for the construction of a prototype for a pneumatic actuator, inspired by the movement of Mimosa pudica L., specific tests and simulations were not carried out. However, with the concepts developed in this study it is possible to base future work on this research for the improvement of pneumatic actuators that use resources such as biomimetics, soft robots and 3D printing, so that we provide insights for improvements and optimizations in this model and other bio-inspired innovations in different areas of technology.

Furthermore, regarding studies of the Mimosa pudica L., there is a lack of studies regarding modeling and simulations from an engineering point of view to support the project in depth. Therefore, the present study was established in the field of biomimetic considering an analysis of the behavior of the plant for the design of structures abstracted from the original shape of the pulvino. The authors' main goal was to characterize the concept used in the movement of the plant (migration of water flow through the xylem vessels and swelling of the cells of the motor organ) and integrate it using engineering concepts. It was achieved through the formulation of conceptual design presented with pneumatic vessels capable of migrating the airflow to the flexible actuator modules (related to the abstraction of the main pulvino) delivering omnidirectional movement to the structure.

\section{CONCLUSION}

Mimosa pudica L., despite the different studies related to the understanding of the functioning of its movement mechanism, presents a behavior capable of acting as an embryo in the projection of new technologies in the field of biomimetics. However, further studies are still necessary for a correct understanding of its functioning, in order to subsidize research for other fields of scientific interest, such as sensing devices and bioinspired materials. In addition, areas such as soft robotics may benefit from such studies, since, by employing fluidic systems and exhibiting properties of soft materials, such as plant tissues, they are capable of being applied in the context of new technologies and applications as areas of automation, robotics and materials science.

Here, the authors presented a conceptual design of an actuator, considering the whole bioinspired design process and its iterations. The final concept could still be improved based on the application it is aimed in future work. Possible improvements would consider the use of fluid control mechanisms with a pneumatic system that works with higher pressures and under more precise controls for the movement of the model. In addition, robotic systems and claw mechanisms could be implemented in the structure in order to develop new actuators made of low-cost materials capable of handling objects in production lines or other means.

Funding: This research was funded by the Scientific Initiation Program (PIC) of the Federal University of ABC - Notice 01/2018 and by FAPESP grant \# 2019/14099-5.

Acknowledgments: This work was supported by the "4D Printing and Biomimetics" (4DB) Research Group - a National Council for Scientific and Technological Development $(\mathrm{CNPq})$ research group at the Federal University of $A B C$ (UFABC).

Conflicts of Interest: The authors declare no conflict of interest.

\section{REFERENCES}

1. Fratzl P, Elbaum R, Burgert I. Cellulose fibrils direct plant organ movements. Faraday discussions. 2008;139:27582.

2. Taya M. Bioinspired design of intelligent materials. Smart Structures and Materials 2003: Electroactive Polymer Actuators and Devices (EAPAD). International Society for Optics and Photonics. 2003;5051:54-65.

3. Shahinpoor M, Thompson MS. The Venus Flytrap as a model for a biomimetic material with built-in sensors and actuators. Materials Science and Engineering: C. 1995 Sep;2(4):229-233.

4. Titotto S. Models and prototypes of biomimetic devices to architectural purposes. SCIRES-IT-SClentific RESearch and Information Technology. 2014;4(2):55-62.

5. Kumar N, Kaur P, Das K, Chakroborty S. Mimosa pudica L. a sensitive plant. Int. J. Pharm. Pharm. Sci. 2009 OctDec;1(2):1-7.

6. Chua CL, Chollet F, He J. Study of a biological actuator and sensor: The Mimosa pudica. Int. J. Comput. Eng. Sci. 2003;4(3):559-562.

7. Volkov AG, Foster JC, Ashby TA, Walker RK, Johnson JA, Markin VS. Mimosa pudica: electrical and mechanical stimulation of plant movements. Plant, cell \& environment. 2010 Jan 11;33(2):163-173.

8. Brattland V, Austvoll I, Ruoff P, Drengstig T. Image Processing of Leaf Movements in Mimosa pudica. In: Sharma P, Bianchi F, editors. Image Analysis. SCIA 2017. Lecture Notes in Computer Science, vol 10269. Springer, Cham; 2017. p. 77-78; 
9. Ahmad H, Sehgal S, Mishra A, Gupta R. Mimosa pudica L. (Laajvanti): an overview. Pharmacognosy reviews. 2012 Jul-Dec;6(12):115-124.

10. Fleurat-Lessard P, Roblin G. Comparative Histocytology of the Petiole and the Main Pulvinus in Mimosa pudica L. Annals of Botany. $1982 \mathrm{Jul} ; 50(1): 83-92$.

11. Fromm J, Eschrich W. Transport processes in stimulated and non-stimulated leaves of Mimosa pudica. Trees. 1988 Mar;2(1):7-17.

12. Lee SJ, Song K, Kim HK, Park J. X-ray CT and histological imaging of xylem vessels organization in Mimosa pudica. Microscopy research and technique. 2013 Sep 12;76(11):1204-1212.

13. Fleurat-Lessard P. Structural and ultrastructural features of cortical cells in motor organs of sensitive plants. Biol. Rev. 1988 Feb;63(1):1-22.

14. Roblin G. Mimosa pudica: a model for the study of the excitability in plants. Biol. Rev. 1979;54(2):135-153.

15. Kagawa H, Saito E. A model on the main pulvinus movement of Mimosa pudica. JSME International Journal Series C Mechanical Systems, Machine Elements and Manufacturing. 2000;43(4):923-8.

16. Song K, Yeom E, Lee SJ. Real-time imaging of pulvinus bending in Mimosa pudica. Scientific Reports. 2014;4(1):18.

17. Malone M. Wound-induced hydraulic signals and stimulus transmission in Mimosa pudica L. New Phytologist. 1994 Sep;128(1):49-56.

18. Guo Q, Dai E, Han X, Xie S, Chao E, Chen Z. Fast nastic motion of plants and bioinspired structures. J. R. Soc. Interface. 2015 Sep 06;12(110):20150598.

19. Kwan KW, Ye ZW, Chye ML, Ngan AH. A Mathematical Model on Water Redistribution Mechanism of the Seismonastic Movement of Mimosa Pudica. Biophys. J. 2013 Jul 2;105(1):266-275.

20. Ismarrubie ZN, Goh HL, Masuri S, Yussof H. Bio-Mechanism Response of Mimosa Pudica against External Stimulation. In: Advanced Materials Research. Trans Tech Publications. 2015 Mar 22;1125:588-592.

21. Lü C, Chen W, Zhou J, Qu S, Chen W. Mechanics of soft materials, structures and systems. Theor. Appl. Mech. Lett. 2013;3(5):2-054001.

22. Wong WS, Li M, Nisbet DR, Craig VS, Wang Z, Tricoli A. Mimosa Origami: A nanostructure-enabled directional selforganization regime of materials. Sci. Adv. 2016 Jun 24;2(6):1-9.

23. Lee H, Xia C, Fang NX. Biomimetic Microactuator Powered by Polymer Swelling. In: ASME 2008 International Mechanical Engineering Congress and Exposition. American Society of Mechanical Engineers; 2008. p. 765-769.

24. Wang G, Cheng T, Do Y, Yang H, Tao Y, Gu J, et al. Printed Paper Actuator: A Low-cost Reversible Actuation and Sensing Method for Shape Changing Interfaces. In: Proceedings of the $2018 \mathrm{CHI}$ Conference on Human Factors in Computing Systems. ACM, 2018. p. 569.

25. Kim S, Laschi C, Trimmer B. Soft robotics: a bioinspired evolution in robotics. Trends in biotechnology; 2013 May;31(5):287-94.

26. Gupta D. Octobot - An entirely soft and autonomous robot. Int. J. Adv. Sci. Res. 2016 Sep;1(6):4-8.

27. Laschi C, Rossiter J, lida F, Cianchetti M, Margheri L. Soft Robotics: Trends, Applications and Challenges. Vol. 17. Springer, 2017.

28. Jiang H, Liu X, Chen X, Wang Z, Jin Y, Chen X. Design and simulation analysis of a soft manipulator based on honeycomb pneumatic networks. In: 2016 IEEE International Conference on Robotics and Biomimetics (ROBIO); 2016 Dec 3-7; Qingdao, China. IEEE Xplore; 2017. p.350-356.

29. Zou J, Lin Y, Ji C, Yang H. A reconfigurable omnidirectional soft robot based on caterpillar locomotion. Soft robotics. 2018 Apr 1;5(2):164-174.

30. Shepherd RF, llievski F, Choi W, Morin SA, Stokes AA, Mazzeo AD, et al. Multigait soft robot. Proceedings of the national academy of sciences. 2011 Dec 20;108(51):20400-3.

31. Soft Robots Toolkit, 2013. Variation: Material. Available from: <https://softroboticstoolkit.com/book/pneunetsvariation-material> [Accessed 24th May 2019].

2021 by the authors. Submitted for possible open access publication under the terms and conditions of the Creative Commons Attribution (CC BY NC) license (https://creativecommons.org/licenses/by-nc/4.0/). 